\title{
Conus medullaris ganglioneuroma with syringomyelia radiologically mimicking ependymoma: A case report
}

\author{
$\mathrm{KAI} \mathrm{WANG}^{1}$ and JIANPING DAI ${ }^{2}$ \\ ${ }^{1}$ Department of Neuroradiology, Beijing Tian Tan Hospital; ${ }^{2}$ Department of Neuroradiology, \\ Beijing Neurosurgical Institute, Capital Medical University, Beijing 100050, P.R. China
}

Received November 15, 2014; Accepted October 1, 2015

DOI: $10.3892 / \mathrm{ol} .2015 .3815$

\begin{abstract}
Ganglioneuromasarerare,benign,well-differentiated tumors of the conus medullaris. Approximately 20 cases of spinal cord ganglioneuroma, and only 1 case of mixed chemodectoma-ganglioneuroma of the conus medullaris have been previously reported. The present study presents the case of a 38-year-old man with a histopathological diagnosis of conus medullaris ganglioneuroma. The patient presented with hypoesthesia in the lower limbs, muscle atrophy of the right lower limb and dysuria. Magnetic resonance imaging analysis led to a diagnosis of ependymoma. Histopathological analysis of the excised mass revealed typical, well-differentiated ganglion cells, consistent with a ganglioneuroma. The mass was associated with a neighboring syringomyelia. At an 18 month follow-up the patient had recovered, although some remaining difficulty in walking and urinating remained. The aim of the present report was to raise awareness that when ganglioneuromas present in unusual locations, analogous radiological findings may mislead investigators to consider more common pathologies and thus result in misdiagnosis. The present case demonstrates the importance of considering the potential differential diagnoses for neural tissue neoplasms.
\end{abstract}

\section{Introduction}

Ganglioneuromas are a type of benign peripheral nerve tumor with an incidence of 1/100,000 (1,2). Furthermore, women are more frequently affected than men (1-3). Ganglioneuromas most frequently occur in the sympathetic ganglia and adrenal medulla, exerting the majority of their effect on spinal and sympathetic nerves, and causing neural dysfunction (4). These benign tumors contain well-differentiated ganglion cells, which are otherwise common in the posterior mediastinum

Correspondence to: Professor Jianping Dai, Department of Neuroradiology, Beijing Neurosurgical Institute, Capital Medical University, 6 Tiantanxili, Beijing 100050, P.R. China

E-mail: djpbeijing@hotmail.com

Key words: ganglioneuroma, conus medullaris, syringomyelia, magnetic resonance imaging and retroperitoneal regions. Ganglioneuromas located in the spinal cord are rare, and are frequently dumbbell-shaped $(5,6)$. Ganglioneuromas typically present at a late stage due to their slow growth. Recurrence and malignant transformation of the tumor are rare, thus, complete surgical excision provides effective cure (7). When present in uncommon locations, such as the cauda equina, incorrect provisional diagnoses of ganglioneuroma, as other more common neoplasms, are likely, due to its analogous radiological features (8). For this reason, it is important to maintain a broad list of differential diagnoses until histopathological findings can confirm a diagnosis. To date, only one mixed chemodectoma-ganglioneuroma of the conus medullaris region has been reported (9). The current study reports a case of ganglioneuroma located at the conus medullaris. The patient underwent laminotomy surgery and a favorable outcome was achieved. Written informed consent was obtained from the patient.

\section{Case report}

A 38-year-old Chinese man presented to Beijing Tian Tin Hospital (Beijing, China) in October 2012, with an 8-month history of numbness in the lower limbs and muscle atrophy of the right lower limb. The patient had also experienced dysuria over the preceding month. Routine physical examination revealed no notable abnormalities. Neurological examination confirmed hypoesthesia of the hypogastrium region and the two lower limbs, grade IV myodynamia of the lower limbs and grade $\mathrm{V}$ myodynamia of the upper limbs (10). Family history revealed no indication of a hereditary etiology. Routine blood, urine, blood biochemistry and coagulation function tests were normal.

Spinal magnetic resonance imaging (MRI) revealed an irregular-shaped mass $(1.5 \times 1.0 \times 1.0 \mathrm{~cm})$ located in the conus medullaris (Fig. 1). The major section of the mass appeared isointense on T1-weighted (T1WI) and T2-weighted (T2WI) images. At the inferior pole of the lesion, there was a nodular, low-intensity signal identified by T1WI and T2WI scans. The conus medullaris surface revealed a discontinuous punctiform or linear high-intensity signal on the T1WI. On the contrast-enhanced T1WI, the major portion was homogeneously enhanced and the conus medullaris surface showed a visible linear enhancement compared with that of the T1WI. Furthermore, at the upper end of the lesion, a large syringomyelia 


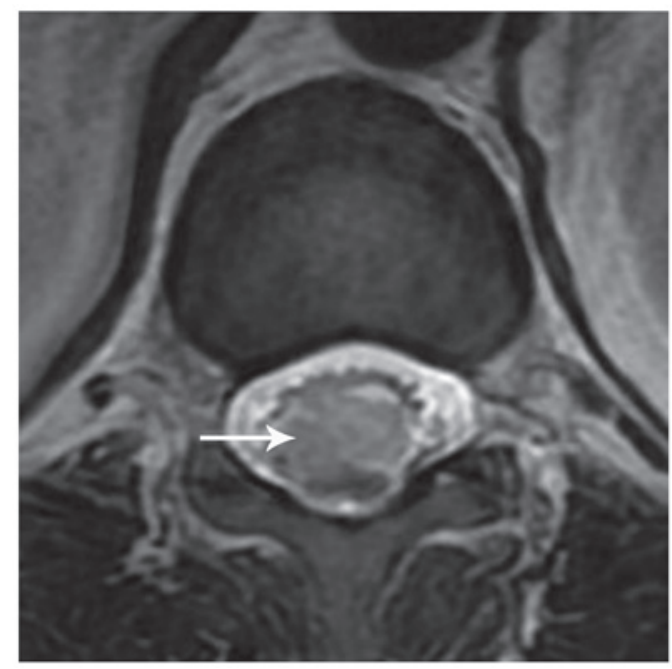

Figure 1. Axial T2WI scan of the spine prior to surgery. An irregular-shaped, isointense mass (arrow) was identified in the conus medullaris.
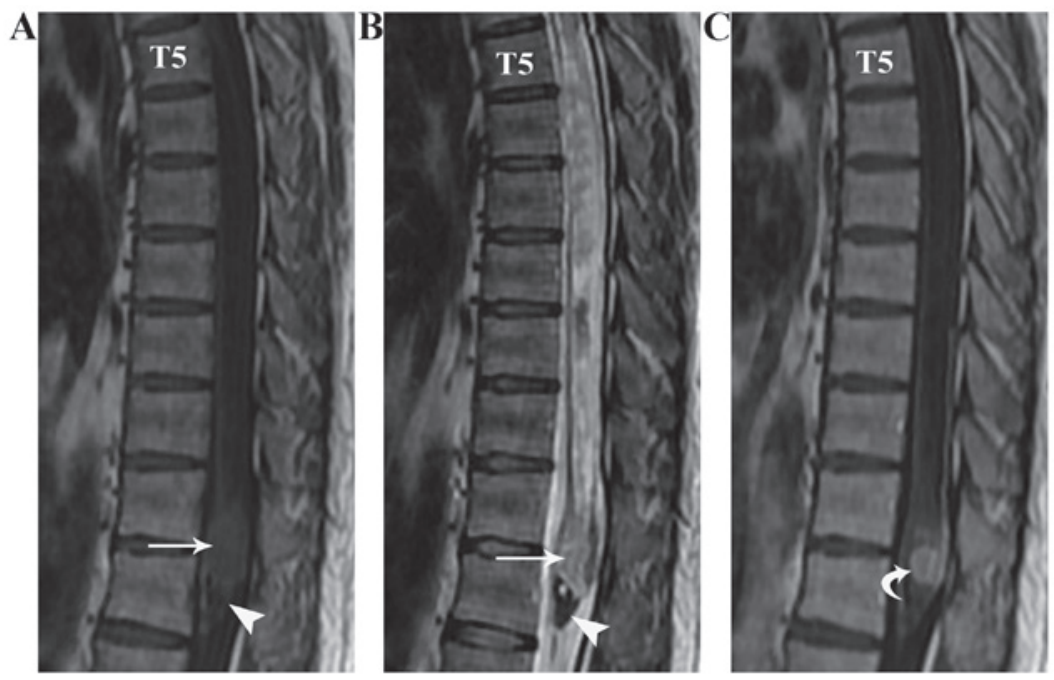

Figure 2. Sagittal (A) T1WI, (B) T2WI and (C) contrast-enhanced T1WI of the spine prior to surgery. The major portion of the tumor (arrow) appeared as an isointensity on T1WI and T2WI. A nodular, low-intensity signal was detected on T1WI and T2WI at the inferior pole of the tumor (arrowhead). T1WI revealed a large syringomyelia (T5-11) as a discontinuous punctiform or linear high-intensity signal on the conus medullaris surface. Following contrast injection, the major portion of the tumor demonstrated marked and homogeneous enhancement (curved arrow), while the conus medullaris surface showed linear enhancement.

(T5-11) was identified (Fig. 2). Based on the provisional diagnosis of ependymoma, surgery was undertaken to excise the mass. During the laminotomy, surgeons incised the dura mater along the posterior midline to expose the lesion and the tumor was visually confirmed to lie in the conus medullaris. The tumor was gray-red and rubbery, with a blood supply and a draining vein in the rostral pole. Complete resection was performed.

Histological examination revealed that the neoplastic lesion consisted of spindle-shaped cells, between which, large ganglion cells with vesicular nuclei and eosinophilic nucleoli were identified (Fig. 3). Immunohistochemical staining was positive for $\mathrm{S}-100$, and the neuronal markers synaptophysin (SYN), neuron-specific enolase (NSE), microtubule-associated protein 2 (MAP-2) and neurofilament (NF). Staining was negative for glial fibrillary acidic protein (GFAP), NeuN and myelin basic protein (MBP). Furthermore, the number of cells immunopositive for the cell proliferation marker Ki-67 was $<2 \%$, confirming the benign nature of the mass.

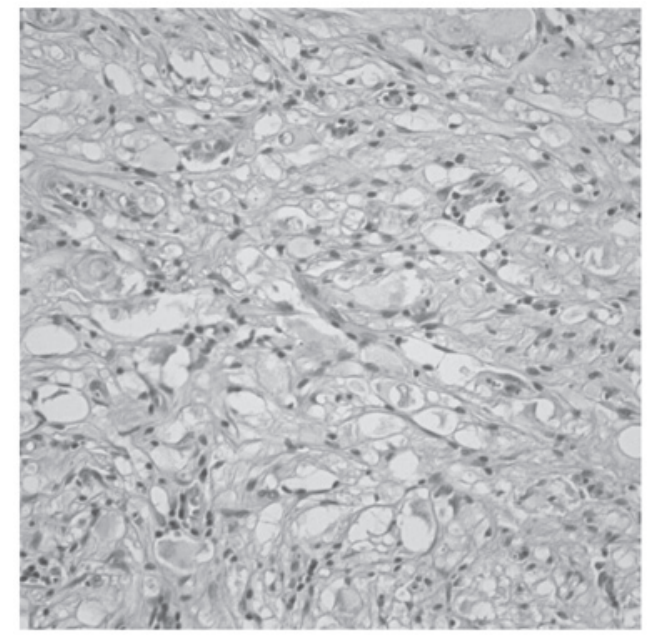

Figure 3. Hematoxylin and eosin staining photomicrograph (magnification, $\mathrm{x} 20$ ). Postoperative histopathology of the excised tumor revealed ganglion cells with vesicular nuclei and eosinophilic nucleoli. 

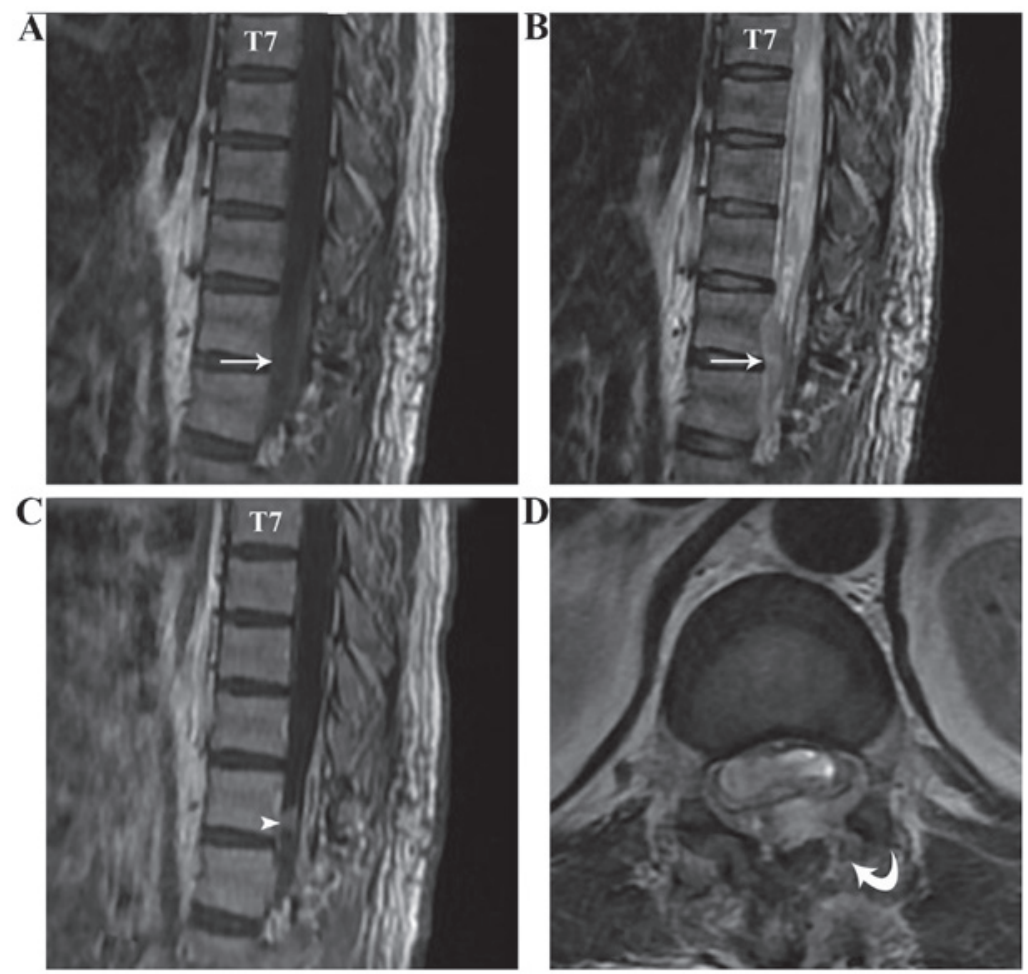

Figure 4. Sagittal (A) T1WI, (B) T2WI, (C) contrast-enhanced T1WI and (D) axial T2WI of the spine following surgery. The tumor was excised (arrow), revealing lamina discontinuity (curved arrow). Following contraast injection, there was a nodular, enhanced signal within the operation scope (arrowhead).

MRI examination 3 days subsequent to surgery confirmed that the tumor was excised and there was no notable syrinx collapse or retraction of the spinal cord (Fig. 4). The patient was discharged on the ninth post-operative day. At the follow-up consultation 18 months later, the patient exhibited moderate difficulty walking and urinating, but was otherwise well.

\section{Discussion}

Derived from the embryonic neural crest, ganglioneuromas arise from sympathoblasts and mainly occur in the sympathetic ganglion and adrenal medulla, common particularly in the posterior mediastinum and retroperitoneal region (11). The explanation for the uncommon location of ganglioneuroma is likely a result of the mechanism of neural crest cell migration in the embryonic period (4). The neural crest consists of a band of longitudinal cells between the neural tube and epidermis, when the neural tube is completed (12). Subsequently, sections of the neural crest cells migrate to the ventral side, while certain cells remain in the neural tube (spinal cord), when the neural crest is formed at the back of neural tube $(13,14)$. Therefore, the conus medullaris region is a possible anatomical location for the origin of ganglioneuroma $(4,12-15)$.

Ganglioneuroma and ganglioneuroblastomas are types of neuroblastoma $(15,16)$. Ganglioneuromas are frequently localized along the sympathetic chain in the posterior mediastinal, retroperitoneal, adrenal gland and cervical spinal regions $(17,18)$. Other potential locations include the presacrum, thoracolumbar region and bone $(19,20)$. Ganglioneuromas located in the conus medullaris, and associated with syringomyelia, are rare. In the presence of a syringomyelia, or when a low-intensity signal is present on T2WI scans (indicating calcified foci or hemorrhage), an incorrect diagnosis of ependymoma may be made. On occasion, the features of ganglioneuromas may mimic those of myxopapillary ependymomas, which are often located in the conus medullaris and the cauda equina, and are commonly associated with hemorrhages that appear as low-intensity signals on T2WI $(9,21)$. Other spinal lesions, for example hemangioblastomas, which are characterized by small lesions with multi-sectional syringomyelia, are also difficult to differentiate (22). Similarly, in the present case, a contrast-enhanced vessel was identified above the lesion in the T1WI. Differential diagnosis is also difficult with spinal paragangliomas, where the tumor is always in the cauda equina, showing intense homogeneous enhancement by MRI, whereas large paragangliomas may show bony remodeling or erosion $(23,24)$. For these reasons a radiological diagnosis based on such analogous imaging features is difficult and may result in misdiagnosis.

Pathologically, ganglioneuromas are well-differentiated, benign tumors, which are populated by mature sympathetic ganglion cells (17). Typically, such features may aid the distinction of these tumors from other spinal lesions, including ependymomas, hemangioblastomas, paragangliomas, schwannomas and neurofibromas. Further histological examination in the present study revealed that the neoplastic lesion consisted of spindle-shaped cells, between which, large ganglion cells with vesicular nuclei and eosinophilic nucleoli were found. The ganglion cells were positively stained for S-100, SYN, NSE, MAP-2 and NF.

During surgery, the tumor was completely resected and the associated syringomyelia did not receive any specific treatment. The tumor-associated cyst or syrinx is typically expected to shrink, or even fully return to normal, following tumor 
resection. However, post-operative MRI indicated no marked syrinx collapse or retraction of the spinal cord. At the 18-month follow-up, the patient still exhibited mild motor deficits. It was therefore hypothesized that the pre-existing syringomyelia may have been the reason underlying this long-term damage (25) Pressure on spinal cord parenchyma, secondary to syringomyelia cavity expansion, may have contributed to the spinal cord dysfunction, particularly since intra-syrinx fluid is typically characterized by high viscosity and pressure (26). While it has not been studied extensively, it has been suggested that spinal cord ischemia may be another significant contributor to spinal cord dysfunction. Ischemia may arise from a marked increase in regional spinal cord blood flow following decompression of the syrinx $(27,28)$. Damage sustained in this way is likely to contribute to compromised post-operative spinal cord function and sub-optimal recovery from surgical resection.

In conclusion, ganglioneuromas are benign, well-differentiated tumors. When present in uncommon locations, for example the cauda equina, incorrect provisional diagnoses of other, more common neoplasms are likely due to the analogous radiological features. For this reason, it is important to maintain a broad range of differential diagnoses until histopathological findings are able to confirm a diagnosis.

\section{References}

1. Mounasamy V, Thacker MM, Humble S, Azouz ME, Pitcher JD, Scully SP, Temple HT and Eismont F: Ganglioneuromas of the sacrum - a report of two cases with radiologic-pathologic correlation. Skeletal Radiol 35: 117-121, 2006.

2. Modha A, Paty P and Bilsky MH: Presacral ganglioneuromas. Report of five cases and review of the literature. J Neurosurg Spine 2: 366-371, 2005.

3. MacCarty CS, Waugh JM, Coventry MB and Cope WF Jr: Surgical treatment of sacral and presacral tumors other than sacrococcygeal chordoma. J Neurosurg 22: 458-464, 1965.

4. Spinelli C, Rossi L, Barbetta A, Ugolini C and Strambi S: Incidental ganglioneuromas: A presentation of 14 surgical cases and literature review. J Endocrinol Invest 38: 547-554, 2015.

5. Shephard RH and Sutton D: Dumb-bell ganglioneuromata of the spine with a report of four cases. Br J Surg 45: 305-317, 1958

6. Oro JJ and Geise AW: Dumbbell ganglioneuroma of the lumbar spine associated with a herniated intervertebral disc: Case report. Neurosurgery 13: 711-714, 1983.

7. Li L, Shao J, Gu J, Wang X and Qu L: Adrenal ganglioneuromas: Experience from a retrospective study in a Chinese population. Urol J 11: 1485-1490, 2014

8. Okudera Y, Miyakoshi N, Sugawara T, Hongo M, Kasukawa Y, Ishikawa Y and Shimada Y: Ganglioneuroblastoma of filum terminale: Case report. J Neurosurg Spine 21: 270-274, 2014.

9. Schmitt HP, Wurster K, Bauer M and Parsch K: Mixed chemodectoma-ganglioneuroma of the conus medullaris region. Acta Neuropathol 57: 275-281, 1982.
10. Liu WH and Du YH: Therapeutic observation of needling three hand yang meridian acupoints to treat postapoplectic finger contracture. J Acupunct Tuina Sci 5: 301-303, 2007.

11. Kyoshima K, Sakai K, Kanaji M, Oikawa S, Kobayashi S, Sato A and Nakayama J: Symmetric dumbbell ganglioneuromas of bilateral $\mathrm{C} 2$ and $\mathrm{C} 3$ roots with intradural extension associated with von Recklinghausen's disease: Case report. Surg Neurol 61: 468-473, 2004.

12. Geoerger B, Hero B, Harms D, Grebe J, Scheidhauer K and Berthold F: Metabolic activity and clinical features of primary ganglioneuromas. Cancer 91: 1905-1913, 2001.

13. Dimou J, Russell JH, Jithoo R and Pitcher M: Sacral ganglioneuroma in a 19-year-old woman. J Clin Neurosci 16: 1692-1694, 2009.

14. Ma J, Liang L and Liu H: Multiple cervical ganglioneuroma: A case report and review of the literature. Oncol Lett 4: 509-512, 2012.

15. Hayes FA, Green AA and Rao BN: Clinical manifestations of ganglioneuroma. Cancer 63: 1211-1214, 1989.

16. Esiri M: Russell and Rubinstein's pathology of tumors of the nervous system. Sixth edition. J Neurol Neurosurg Psychiatry 68: 538D, 2000.

17. Ghali VS, Gold JE, Vincent RA and Cosgrove JM: Malignant peripheral nerve sheath tumor arising spontaneously from retroperitoneal ganglioneuroma: A case report, review of the literature and immunohistochemical study. Hum Pathol 23: 72-75, 1992.

18. Andersen HJ, Hansen LG, Lange P and Teglbjaerg PS: Presacral ganglioneuroma. Case report. Acta Chir Scand 152: 777-778, 1986.

19. Levy DI, Bucci MN, Weatherbee L and Chandler WF: Intradural extramedullary ganglioneuroma: Case report and review of the literature. Surg Neurol 37: 216-218, 1992.

20. Cerullo G, Marrelli D, Rampone B, Miracco C, Caruso S, Di Martino M, Mazzei MA and Roviello F: Presacral ganglioneuroma: A case report and review of literature. World J Gastroenterol 13: 2129-2131, 2007.

21. Kariev GM, Halikulov ES and Rasulov SO: Unspecific clinical manifestation of cauda equina myxopapillary ependymoma. Asian J Neurosurg 10: 256-258, 2015.

22. Deng X, Wang K, Wu L, Yang C, Yang T, Zhao L, Yang J, Wang $\mathrm{G}$, Fang $\mathrm{J}$ and $\mathrm{Xu} \mathrm{Y}$ : Intraspinal hemangioblastomas: analysis of 92 cases in a single institution: Clinical article. J Neurosurg Spine 21: 260-269, 2014.

23. Lmejjati M, Parker F, Lacroix C and Tadie M: Paraganglioma of the sacral spinal canal. Neurosciences (Riyadh) 16: 270-272, 2011.

24. Midi A, Yener AN, Sav A and Cubuk R: Cauda equina paraganglioma with ependymoma-like histology: A case report. Turk Neurosurg 22: 353-359, 2012.

25. Young WF, Tuma R and O'Grady T: Intraoperative measurement of spinal cord blood flow in syringomyelia. Clin Neurol Neurosurg 102: 119-123, 2000.

26. Klekamp J: The pathophysiology of syringomyelia - historical overview and current concept. Acta Neurochir (Wien) 144: 649-664, 2002.

27. Levine DN: The pathogenesis of syringomyelia associated with lesions at the foramen magnum: A critical review of existing theories and proposal of a new hypothesis. J Neurol Sci 220: 3-21, 2004.

28. Schaller B, Mindermann T and Gratzl O: Treatment of syringomyelia after posttraumatic paraparesis or tetraparesis. J Spinal Disord 12: 485-488, 1999. 